\title{
Use of Unamplified RNA/cDNA-Hybrid Nanopore Sequencing for Rapid Detection and Characterization of RNA Viruses
}

\author{
Andy Kilianski, Pierce A. Roth, Alvin T. Liem, \\ Jessica M. Hill, Kristen L. Willis, \\ Rebecca D. Rossmaier, Andrew V. Marinich, \\ Michele N. Maughan, Mark A. Karavis, \\ Jens H. Kuhn, Anna N. Honko, \\ C. Nicole Rosenzweig
}

Nanopore sequencing, a novel genomics technology, has potential applications for routine biosurveillance, clinical diagnosis, and outbreak investigation of virus infections. Using rapid sequencing of unamplified RNA/cDNA hybrids, we identified Venezuelan equine encephalitis virus and Ebola virus in 3 hours from sample receipt to data acquisition, demonstrating a fieldable technique for RNA virus characterization.

$\mathrm{P}$ ortable and reliable molecular epidemiology techniques and field approaches for assessing virus genomes are desired to inform clinical diagnostics and public health operations. Need for such methods has been highlighted by the recent Middle East respiratory syndrome and Ebola virus disease (EVD) epidemics, during which it became necessary to characterize novel viruses and to evaluate genetic drift, transmission chains, and zoonotic introductions.

To determine if nanopore sequencing can be used as an accelerated viral genome sequencing tool, we utilized a rapid cDNA/RNA-hybrid library preparation procedure to sequence cell cultures of Venezuelan equine encephalitis virus vaccine (VEEV) strain TC-83 or Ebola virus (EBOV) isolate Makona-C05 stock IRF0137. To evaluate nanopore sequencing for rapid, field-deployable pathogen characterization, we collected raw read data and statistics for VEEV and EBOV sequence runs on the MinION sequencing device (Oxford Nanopore Technologies, Oxford, UK). To determine the level of identification and accuracy of genome characterization over sequencing runtime, these

Author affiliations: US Army Edgewood Chemical Biological Center, Aberdeen Proving Ground, Maryland, USA

(A. Kilianski, P.A. Roth, A.T. Liem, J.M. Hill, K.L. Willis, R.D. Rossmaier, A.V. Marinich, M.N. Maughan, M.A. Karavis, C.N. Rosenzweig); Defense Threat Reduction Agency, Fort Belvoir, Virginia, USA (K.L. Willis); National Institutes of Health, Fort Detrick, Frederick, Maryland, USA (J.H. Kuhn, A.N. Honko)

DOI: http://dx.doi.org/10.3201/eid2208.160270 reads were then mapped to VEEV and EBOV genomes and to reference databases (RefSeq [http://www.ncbi.nlm.nih. gov/RefSeq/]). From the results of these analyses, we determined that the current and future versions of nanopore sequencing technology can be used to rapidly identify and characterize pathogens.

\section{The Study}

This approach for pathogen identification and characterization differs from the previously used methods on the MinION platform. Biased techniques, such as amplicon sequencing, have proven to be effective in complex sample backgrounds in which titers of the target pathogen might be low, but such approaches limit characterization to known pathogens and require additional viral genome amplification (1-4). Unbiased techniques that require viral genome amplification (5) or that have been optimized for bacterial genomes $(6,7)$ require longer sample and library preparation times, but can detect low pathogen titers or create highly accurate genomic data. We sequenced unamplified poly(A)-tailed viral RNA using rapid cDNA library preparation coupled with real-time data analysis to determine its potential application for pathogen genomic characterization.

VEEV has a single-stranded, linear, poly(A)-tailed RNA genome. Thus, poly-dT primers can be used for cDNA production without further genomic RNA manipulation. The workflow to isolate the RNA and prepare it for sequencing (online Technical Appendix, http://wwwnc.cdc. gov/ article/22/8/16-0270-Techapp1.pdf) took $\approx 3$ hours from the initiation of sample processing to data acquisition on MinION (Figure, panel A). The sequencing of VEEV genomic RNA/cDNA hybrids attained in hours by using MinION revealed reads that mapped to the VEEV TC-83 genome within minutes by using the LAST (Computational Biology Research Consortium, Tokyo, Japan) multiple sequence alignment program (online Technical Appendix; Figure, panel B $[2,4,6])$. The coverage increased from 1560 min from the $3^{\prime}$ end of the VEEV genome with reads aligning directionally from the $3^{\prime}$ to $5^{\prime}$ end of the VEEV genome (Figure, panel B). These alignment characteristics are indicative of the poly-dT priming strategy for poly(A)tailed RNA.

To determine if the reads generated from VEEV TC83 would align to the correct viral genome within a set 


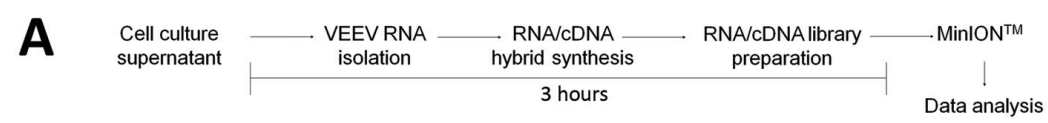

B

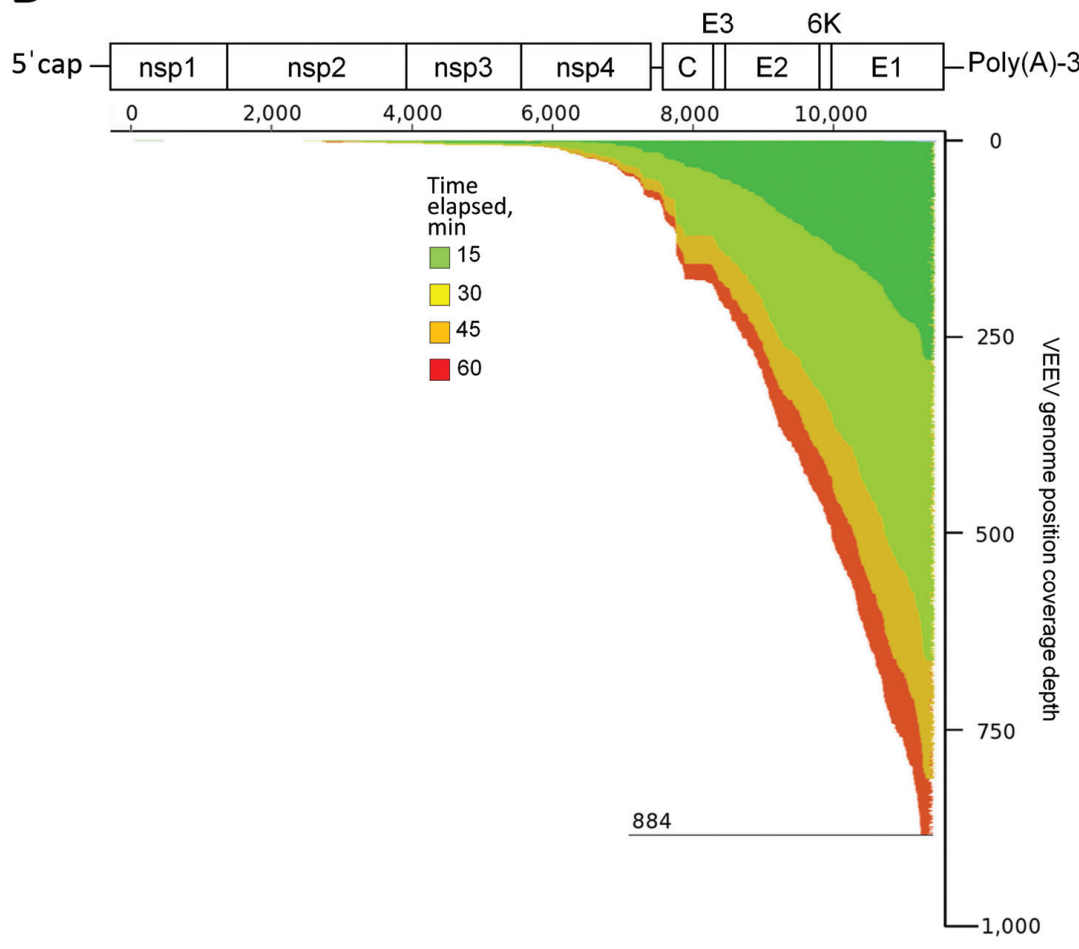

Figure. Use of unamplified RNA/cDNA-hybrid nanopore sequencing for genomic characterization of Venezuelan equine encephalitis virus (VEEV) TC-83. A) Sample preparation workflow for nanopore sequencing. First, viral RNA from BHK21 cell cultures of VEEV TC-83 was isolated, then single strand complimentary DNA (cDNA) was synthesized. The resulting RNA/cDNA hybrids were then prepared for nanopore sequencing and sequenced with data analysis occurring in real time. B) Genome organization and sequencing coverage over time of VEEV TC-83. VEEV is an alphavirus; its genome consists of a single strand of positive-sense RNA that can be translated into a polyprotein. Translation is critically dependent on the genomic 3' poly(A)-tail. This tail can be used for reverse transcription priming by using poly-(dT) primers that anneal to it. Read data were aligned to VEEV TC-83 (accession number L01443) by using the multiple sequence alignment program LAST (Computational Biology Research Consortium, Tokyo, Japan [online Technical Appendix, wwwnc.cdc.gov/ article/22/8/160270-Techapp1.pdf). The coverage map shows the depth of genome coverage over $15,30,45$, and 60 minutes of sequencing runtime, with the greatest depth observed at the $3^{\prime}$ end of the VEEV genome. Nsp, nonstructural protein; $C$, capsid; E, envelope. of reference sequences, we used the viral genome reference sequences (http://www.ncbi.nlm.nih.gov/genomes/ GenomesGroup.cgi?taxid=10239\&opt=Virus), plus the VEEV TC-83 genome database (GenBank accession no. L01443). We then used LAST to align nanopore reads against this set of references (online Technical Appendix). These alignments were used to generate a top hit table and the associated read alignment statistics against each hit. VEEV TC-83 was the top hit based on LAST alignment versus virus RefSeq genome sequences; wildtype VEEV placed second (Table). VEEV TC-83 was also identified as the top hit when the 15- and 60-min sequencing datasets were compared with alphavirus genome sequences (online Technical Appendix) (Table), demonstrating accuracy and depth achieved in a short time. We also analyzed the VEEV TC-83 dataset using the cloud-based metagenomic detection platforms $\mathrm{Pa}-$ thosphere (8) and One Codex (www.onecodex.com), and found that the sample contained VEEV (online Technical Appendix).

Molecular epidemiology, including use of viral genomics, played a major role during the 2013-2016 EVD response, informing contact tracing, diagnostic operability, and public health measures (9-11). To determine if EBOV is amenable to the same rapid sequencing methodology that was used for VEEV, unamplified negative-stranded RNA isolated from EBOV in Trizol (Thermofisher Scientific, http://www.thermofisher.com/us/en/home/brands/productbrand/trizol.html) was poly(A)-tailed, a single complementary strand of cDNA synthesized, and RNA/cDNA hybrids sequenced. The EBOV samples sequenced on MinION rapidly provided usable, accurate data, despite less raw data than the VEEV TC-83 dataset (137kbp for EBOV versus $2.4 \mathrm{Mbp}$ for VEEV at $60 \mathrm{~min}$ ). Using 15 -and 60 -min time points and an identical alignment strategy to VEEV TC-83 above, we detected EBOV as the top hit within the sequencing dataset when compared to all virus RefSeq sequences (Table).

Despite success against the RefSeq database, the lack of depth within the dataset did not enable differentiation between the EBOV isolate sequenced here and the $>1,500$ EBOV draft genomes sequenced during the 2013-2016 outbreak $(10,12,13)$, which indicates a limitation in this sequencing approach for negative-stranded RNA viruses. The poly(A)-tailing method was chosen because the reverse transcription primer adapters designed by Oxford Nanopore were developed to interact directly with the motor protein necessary for guiding DNA through the nanopores. This method greatly reduced preparation time and eliminated need for adaptor ligation reagents. This approach can 
Table. Alignment statistics for detection of VEEV TC-83 and EBOV/Mak-C05 using unamplified RNA/cDNA-hybrid nanopore sequencing*

\begin{tabular}{|c|c|c|c|c|c|c|}
\hline $\begin{array}{l}\text { Virus samples and } \\
\text { time points, min }\end{array}$ & Top hits (GenBank accession no.) & LAST score & $\begin{array}{l}\text { Total bases } \\
\text { mapped, } \%\end{array}$ & $\begin{array}{c}\text { Coverage, } \\
\%\end{array}$ & $\begin{array}{c}\text { Average } \\
\text { base depth }\end{array}$ & $\begin{array}{c}\text { Per read } \\
\text { accuracy, \% }\end{array}$ \\
\hline \multicolumn{7}{|c|}{ VEEV TC-83 (GenBank accession no. L01443) } \\
\hline \multicolumn{7}{|c|}{ Viral genomes (RefSeq databases $†$ ) } \\
\hline \multirow{2}{*}{15} & VEEV TC-83 (L01443) & 138,321 & 5.54 & 76.14 & $50.94 x$ & $59-80$ \\
\hline & VEEV WT (NC 001449.1) & 789 & 0.05 & 18.59 & $1.76 \mathrm{x}$ & $60-78$ \\
\hline \multirow[t]{2}{*}{60} & VEEV TC-83 (L01443) & 419,153 & 17.17 & 78.54 & $153.16 x$ & $57-80$ \\
\hline & VEEV WT (NC_001449.1) & 1,182 & 0.08 & 32.12 & $1.82 x$ & $58-78$ \\
\hline \multicolumn{7}{|c|}{ Alphavirus genomes } \\
\hline \multirow[t]{3}{*}{15} & VEEV TC-83 (L01443) & 31,320 & 1.13 & 48.92 & $16.21 x$ & $67-69$ \\
\hline & VEEV E541/73 (AF093102.1 & 6,463 & 0.27 & 95.07 & $5.26 x$ & $62-73$ \\
\hline & VEEV 71-180 (AF069903.1) & 5,865 & 0.22 & 30.18 & $5.08 \mathrm{x}$ & $65-73$ \\
\hline \multirow[t]{3}{*}{60} & VEEV TC-83 (L01443) & 96,348 & 3.55 & 48.92 & $50.84 x$ & $62-74$ \\
\hline & VEEV E541/73 (AF093102.1) & 21,411 & 0.89 & 99.91 & $16.36 x$ & $61-73$ \\
\hline & VEEV 71-180 (AF069903.1) & 16,429 & 0.64 & 51.04 & $8.78 x$ & $65-73$ \\
\hline \multicolumn{7}{|c|}{ EBOV/Mak-C05 (GenBank accession no. KX000400) } \\
\hline \multicolumn{7}{|c|}{ Viral genomes (RefSeq databases) } \\
\hline \multirow[t]{2}{*}{15} & EBOV/Mak-137 (KX000400) & 529 & 0.11 & 9.29 & $1.00 x$ & 68 \\
\hline & Bovine herpesvirus (NC_024303.1) & 73 & 0.02 & 0.18 & $1.00 \mathrm{x}$ & 67 \\
\hline \multirow[t]{2}{*}{60} & EBOV/Mak-137 (KX000400) & 2,371 & 0.53 & 22.23 & $2.09 x$ & $66-71$ \\
\hline & Bovine herpesvirus (NC 024303.1) & 239 & 0.04 & 0.27 & $1.58 \mathrm{x}$ & $67-74$ \\
\hline
\end{tabular}

*VEEV, Venezuelan equine encephalitis virus; EBOV, Ebola virus; LAST (Computational Biology Research Consortium, Tokyo, Japan), multiple sequence alignment program.

†RefSeq, NCBI Reference Sequence Database (http://www.ncbi.nlm.nih.gov/RefSeq/).

be revisited for sequencing negative-strand RNA viruses $(2,5)$. Despite this limitation, the RefSeq alignments and nearest neighbor calls were possible with limited data, demonstrating the potential power of long-read rapid sequencing on nanopore platforms.

\section{Conclusions}

The current Middle East respiratory syndrome, EVD, and Zika virus disease outbreaks illustrate the necessity for rapid characterization of pathogens for environmental detection, clinical evaluation, and epidemiologic investigation. To determine whether nanopore sequencing can fill this role in a fieldable platform, we tested an RNA/cDNAhybrid sequencing approach on VEEV TC-83 (a positivestranded RNA virus) and EBOV (a negative-stranded RNA virus) prepared from cell-culture supernatants. This method definitively identified VEEV TC-83 and differentiated it from wild-type VEEV in $\approx 3$ hours, including only $15 \mathrm{~min}$ of data acquisition on MinION. VEEV TC-83 was also differentiated from other alphavirus genomes, facilitating strain-level identification of TC-83. EBOV was also identified rapidly by this approach, differentiating the virus in the sample analyzed here from available virus reference genomes. However, variant/isolate level characterization was not possible due to limited data generated from the RNA/ cDNA-hybrid approach.

The method applied here is greatly accelerated compared to traditional next-generation sequencing library preparation, and was used with reagents and equipment suitable for austere conditions (e.g., little need for cold chain, steps not requiring PCR). This study confirmed the possibility of accurate RNA virus genome characterization from RNA/cDNA hybrids by using limited sample manipulation, albeit from relatively pure samples. If samples derived directly from clinical matrices (e.g., blood, saliva) were used, this method would probably not support the necessary depth to characterize virus genomes unless the pathogen titer within these samples was high. As the depth of sequence data obtained from nanopore sequencing approaches continues to improve (14) and other pore types (such as RNA-specific sequencing pores) are integrated into commercial products, these unamplified techniques can transition from the laboratory to the field for more complex analysis.

Utilization of nanopore sequencing in Western Africa $(2,3)$ has demonstrated potential for its use, and newly developed methods like this RNA/cDNA-hybrid approach can be integrated into fieldable protocols. For the emerging Zika virus, insufficiently high virus titers in clinical samples usually necessitates virus culture before genomic sequencing (15). Genomic Zika virus isolate characterization efforts would greatly benefit from the approaches outlined here, especially regarding materials needed for genomic library preparation and the time reduction for strain-level identification (15). By preparing and sequencing RNA/cDNA hybrids, the sampleto-answer time for RNA sequencing is greatly reduced, providing pathogen identification and characterization rapidly to inform future decision making.

Dr. Kilianski is a National Research Council fellow in the BioSciences Division at Edgewood Chemical Biological Center. His research focuses on biosurveillance, emerging viral pathogens, and the identification and characterization of novel agents that threaten today's warfighter. 
The authors acknowledge the efforts of the Sabeti Laboratory and Broad Institute of MIT and Harvard for providing the consensus sequence for EBOV/Mak-C05

This project was funded through the United States Army and Edgewood Chemical Biological Center 219 Innovative Project initiative to A.K. A.K. is also supported by the US National Academy of Science and the US Defense Threat Reduction Agency as a National Research Council fellow. A.K. is part of the Oxford Nanopore MinION Access Program, and has received free materials for this research. This work was funded in part through the prime contract of Battelle Memorial Institute, Columbus, Ohio, with the US National Institute of Allergy and Infectious Diseases under Contract no. HHSN272200700016I. A subcontractor to Battelle Memorial Institute who performed this work is: J.H.K., an employee of Tunnell Government Services, Inc, Bethesda, MD. Information in this report is cleared for public release and distribution is unlimited.

\section{References}

1. Kilianski A, Haas JL, Corriveau EJ, Liem AT, Willis KL, Kadavy DR, et al. Bacterial and viral identification and differentiation by amplicon sequencing on the MinION nanopore sequencer. Gigascience. 2015;4:12. http://dx.doi.org/10.1186/ s13742-015-0051-Z

2. Hoenen T, Groseth A, Rosenke K, Fischer RJ, Hoenen A, Judson SD, et al. Nanopore sequencing as a rapidly deployable Ebola outbreak tool. Emerg Infect Dis. 2016;22:331-4; Epub ahead of print.

3. Quick J, Loman NJ, Duraffour S, Simpson JT, Severi E, Cowley L, et al. Real-time, portable genome sequencing for Ebola surveillance. Nature. 2016;530:228-32. http://dx.doi.org/10.1038/ nature 16996

4. Wang J, Moore NE, Deng Y-M, Eccles DA, Hall RJ. MinION nanopore sequencing of an influenza genome. Front Microbiol. 2015;6:766. http://dx.doi.org/10.3389/fmicb.2015.00766

5. Greninger AL, Naccache SN, Federman S, Yu G, Mbala P, Bres V, et al. Rapid metagenomic identification of viral pathogens in clinical samples by real-time nanopore sequencing analysis. Genome Med. 2015;7:99. http://dx.doi.org/10.1186/s13073-015-0220-9

6. Quick J, Ashton P, Calus S, Chatt C, Gossain S, Hawker J, et al. Rapid draft sequencing and real-time nanopore sequencing in a hospital outbreak of Salmonella. Genome Biol. 2015;16:114. http://dx.doi.org/10.1186/s13059-015-0677-2

7. Ashton PM, Nair S, Dallman T, Rubino S, Rabsch W, Mwaigwisya S, et al. MinION nanopore sequencing identifies the position and structure of a bacterial antibiotic resistance island. Nat Biotechnol. 2014;33:296-300; Epub ahead of print. http://dx.doi.org/10.1038/nbt.3103

8. Kilianski A, Carcel P, Yao S, Roth P, Schulte J, Donarum GB, et al. Pathosphere.org: pathogen detection and characterization through a web-based, open source informatics platform. BMC Bioinformatics. 2015;16:416. http://dx.doi.org/10.1186/ s12859-015-0840-5

9. Mate SE, Kugelman JR, Nyenswah TG, Ladner JT, Wiley MR, Cordier-Lassalle T, et al. Molecular evidence of sexual transmission of Ebola virus. N Engl J Med. 2015;373:2448-54 http://dx.doi.org/26465384

10. Park DJ, Dudas G, Wohl S, Goba A, Whitmer SLM, Andersen KG, et al. Ebola virus epidemiology, transmission, and evolution during seven months in Sierra Leone. Cell. 2015;161:1516-26. http://dx.doi.org/10.1016/j.cell.2015.06.007

11. Kugelman JR, Sanchez-Lockhart M, Andersen KG, Gire S, Park DJ, Sealfon R, et al. Evaluation of the potential impact of Ebola virus genomic drift on the efficacy of sequence-based candidate therapeutics. MBiol. 2015;6. http://dx.doi.org/10.1128/ mBio.02227-14

12. Gire SK, Goba A, Andersen KG, Sealfon RSG, Park DJ, Kanneh L, et al. Genomic surveillance elucidates Ebola virus origin and transmission during the 2014 outbreak. Science. 2014;345:136972. http://dx.doi.org/10.1126/science. 1259657

13. Carroll MW, Matthews DA, Hiscox JA, Elmore MJ, Pollakis G, Rambaut A, et al. Temporal and spatial analysis of the 2014-2015 Ebola virus outbreak in West Africa. Nature. 2015;524:97-101. http://dx.doi.org/10.1038/nature14594

14. Ip CLC, Loose M, Tyson JR, de Cesare M, Brown BL, Jain M, et al. MinION analysis and reference consortium: phase 1 data release and analysis. F1000Research. 2015;4:1075 http://dx.doi.org/10.12688/f1000research.7201.1 PMID: 26834992

15. Faria NR. Azevedo R do S da S, Kraemer MUG, Souza R, Cunha MS, Hill SC, et al. Zika virus in the Americas: early epidemiological and genetic findings. Science. 2016;352:345-9. http://dx.doi.org/10.1126/science.aaf5036

Address for correspondence: C. Nicole Rosenzweig, BioDefense Branch, BioSciences Division, Edgewood Chemical Biological Center, Aberdeen Proving Ground, MD 21010, USA; email: carolyn.n.rosenzweig.civ@mail.mil

\section{SPOTLIGHT}

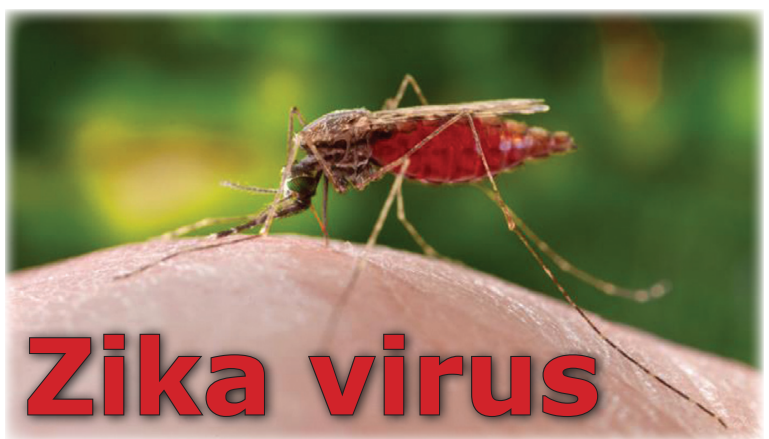

Zika virus is spread to people through mosquito bites. Outbreaks of Zika have occurred in areas of Africa, Southeast Asia, the Pacific Islands, and the Americas. Because the Aedes species mosquitoes that spread Zika virus are found throughout the world, it is likely that outbreaks will spread to new countries. In December 2015, Puerto Rico reported its first confirmed Zika virus case. In May 2015, the Pan American Health Organization issued an alert regarding the first confirmed Zika virus infection in Brazil.

\section{Learn more at http://wwwnc.cdc.gov/eid/page/zika-spotlight}

